\title{
Model Answers to Lipid Membrane Questions
}

\author{
Ole G. Mouritsen \\ MEMPHYS-Center for Biomembrane Physics, Department of Physics and Chemistry, University of \\ Southern Denmark, DK-5230 Odense M, Denmark \\ Correspondence: ogm@memphys.sdu.dk
}

Ever since it was discovered that biological membranes have a core of a bimolecular sheet of lipid molecules, lipid bilayers have been a model laboratory for investigating physicochemical and functional properties of biological membranes. Experimental and theoretical models help the experimental scientist to plan experiments and interpret data. Theoretical models are the theoretical scientist's preferred toys to make contact between membrane theory and experiments. Most importantly, models serve to shape our intuition about which membrane questions are the more fundamental and relevant ones to pursue. Here we review some membrane models for lipid self-assembly, monolayers, bilayers, liposomes, and lipid-protein interactions and illustrate how such models can help answering questions in modern lipid cell biology.

\section{"THEY ARE BAGS!" - A SHORT HISTORY OF MEMBRANE MODELS}

It was a major discovery when Gorter and Grendel in 1925 observed that biological membranes were ultrathin, bimolecular sheets (Gorter and Grendel 1925), thereby suggesting lipid bilayers as simple models of biological membranes (Mouritsen and Andersen 1998). At that time lipid monolayers had already been studied in principle since the days of Benjamin Franklin, and more extensively by Ervin Langmuir and Katharine Blodgett using quantitative experimental techniques. A lipid monolayer at an air-water interface is an even simpler membrane model than a bilayer, basically being half a membrane. Proteins were added to the picture, first peripherally in the 1935 model because of Danielli and Dawson, later in 1966 in Robertson's unit membrane model, and finally in 1972 the Singer-Nicolson fluid mosaic (Singer and Nicolson 1972) anticipated that membranes were composites of a lipid bilayer with integral and membrane-spanning proteins (for a list of references to the classical literature, see the recent review by Bagatolli et al. 2010).

The Singer-Nicolson model was a very successful model because it provided a simple framework within which to ask relevant membrane questions. Its simplicity was its strength, but also its drawback because many researchers, somewhat inadvertently, assumed that membranes were more fluid than mosaic (Engelman 2005). The parallel work on the physical chemistry and phase behavior of lipid bilayer systems during the period 1960-1980 (see, e.g., Shimshick and McConnell 1973) was unfortunately

Editor: Kai Simons

Additional Perspectives on The Biology of Lipids available at www.cshperspectives.org

Copyright (C) 2011 Cold Spring Harbor Laboratory Press; all rights reserved; doi: 10.1101/cshperspect.a004622

Cite this article as Cold Spring Harb Perspect Biol 2011;3:a004622 
only to a minor degree adopted by the biological community (Bergelson 1995). It was only with the introduction of the functional raft concept (Simons and Ikonen 1997; Kusumi and Suzuki 2005; Jacobson et al. 2007; van Meer et al. 2008; Pike 2009; Lingwood and Simons 2010) that the biological researchers became fully aware of the importance of lateral structure and lipid domains in membranes.

The fluid-mosaic model was already significantly refined by Israelachvili (1977) to account for lipid-protein interactions, membrane folding, and pore formation. Further insight into the fundamental mechanisms of membraneprotein interactions was furnished by the Marčelja model (Marčelja 1976) and the so-called mattress model (Mouritsen and Bloom 1984). The mattress model and the principle of triggering mechanisms proposed by Sackmann (Sackmann 1984) both anticipated the importance of differential structuring of membrane regions around integral membrane proteins. Sackmann also refined the model to incorporate the importance of extended cellular structures attached to the membrane, such as the glycocalyx and the cytoskeleton (Sackmann 1995).

A key discovery in biomembrane science is Alec Bangham's discovery of liposomes (Bangham and Horne 1964) showing by electron microscopy that membranes form spontaneously from phospholipids dispersed in water as thin, closed bags of lipid bilayers. The importance for membrane modeling of the insight behind Bangham's statement— "They are bags!" (A Bangham, pers. comm.) —cannot be overestimated because it pinpoints the importance of entropy as a driving force for membrane formation and stability.

Today researchers freely use all these fundamental ideas to help answering membrane questions using a range of membrane models of various complexities. In all cases lipids, lipid self-assembly, lipid bilayers, lipid domains, and lipid functionality have moved center stage (Mouritsen 2005; Heimburg 2007, 2009; Nag 2008). Lipidology and lipidomics have grown to become key sciences in cell biology.

It should be pointed out that the term "model" is used somewhat differently in different communities of researchers. Physical scientists usually think of a useful model, whether it is an experimental model system or a theoretical model, as the simplest possible construction that captures the relevant properties of a given situation. The virtue of this kind of model is to have as little detail as possible. In contrast, life scientists usually insist on having as many details accounted for as possible in a model to be useful and realistic. We shall adopt the physical scientists' ideal of a model in the present paper. It should also be clarified, that theoretical models come in many different flavors. Some models are of the theoretical variety involving a phenomenological construction of a free energy functional for the system formulated in terms of order parameter fields, such as density, composition, order, and curvature. Other models are statistical mechanical models operating with variables that account for some important, molecular degrees of freedom for the molecules. The most refined of such models are those building on the most accurate atomic-scale interatomic potentials available. Less refined models involve some coarse-grained variables that average over many atomic details. In most cases, the statistical mechanical models require computer simulation techniques, such as molecular dynamics, Monte Carlo, or dissipative particle dynamics simulations, for calculating the properties of the models (Merz and Roux 1996; Shillcock 2008; Marrink et al. 2009).

Biomembrane science has now reached a mature state in which it is possible to attach realistic problems and questions to membrane behavior using a combination of experiments, theoretical modeling, and large-scale computer simulation. The picture of membrane modeling advocated in the present paper is based on such a combined approach.

\section{LIPID SELF-ASSEMBLY, CURVATURE, AND LIPID POLYMORPHISM}

Because of their amphiphilic character, lipids self-assemble in water in a variety of different aggregates and extended structures, depending on lipid molecular structure and thermodynamic conditions. The polymorphism of lipids 
Model Answers to Lipid Membrane Questions

and their phase behavior in water which owe so much to the pioneering work by Luzzati (1968) were fully anticipated in the context of biomembranes in a classical paper by Cullis and de Kruijff (1979) and later by Israelachvili introducing a phenomenological packing parameter (Israelachvili 1992). The effective shapes of the molecules were found to control the morphology of the structures being formed (cf. Fig. 1). Molecules of conical shape would stabilize phases with curvature, such as micelles, hexagonal phases, and cubic phases, whereas more cylindrical shapes would predominantly lead to lamellar bilayers (Seddon and Templer 1995).

It is important to note that even if thermodynamic conditions lead to a stable lamellar bilayer this bilayer may suffer from an in-build curvature stress because of a spontaneous curvature of the participating monolayers. This curvature stress can be released globally or even locally by external agents, solutes, and amphiphiles, as well as peptides and proteins leading to destabilizing of the bilayer and/or formation of local defects such as invaginations, buds, or pores.

\section{HALF A MEMBRANE: THE LIPID MONOLAYER}

Lipid monolayers spread on air-water or oilwater interfaces (cf. Fig. 1B) are simple models of lipid membranes. The advantage of such models is that they can easily be studied and manipulated in a Langmuir trough in which thermodynamic relationships between surface tension and surface area can be measured (Dynarowicz-Latka et al. 2001). The in-plane and lateral structure of the monomolecular film, which can consist of lipids, proteins, and lipid-protein recombinants, can readily be investigated by various spectroscopies, such as fluorescence microscopy and Brewster-angle microscopy, by scattering techniques (X-ray and neutron), or by surface-sensitive scanning-probe
A

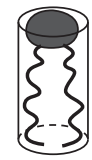

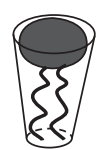

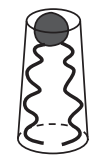

。

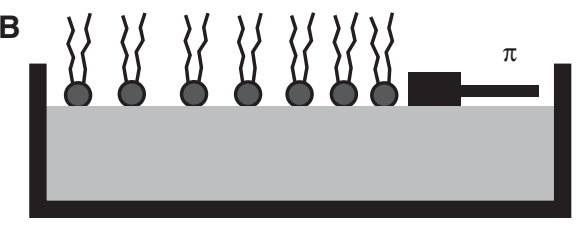

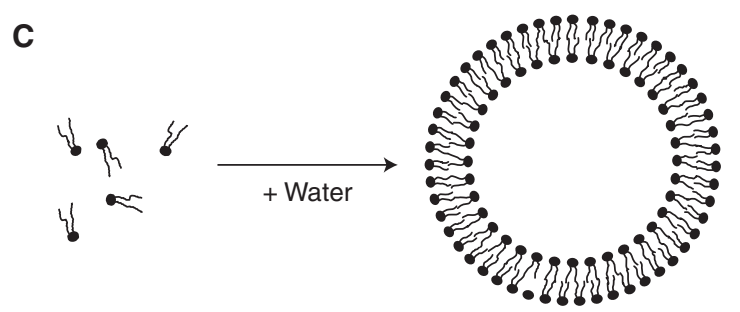

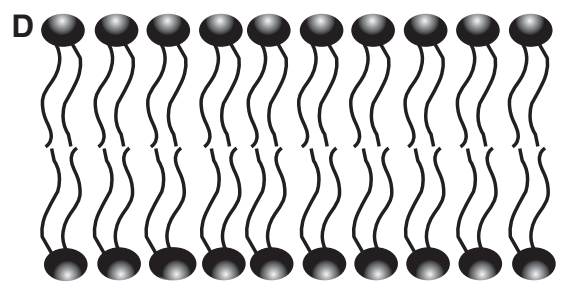

E
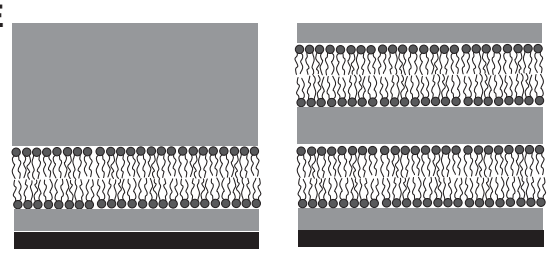

Figure 1. Lipid molecules and models of lipid membranes. (A) Lipid molecular shapes. (B) Lipid monolayer on an air-water interface. $(C)$ Lipid self-assembly into a vesicle (unilamellar liposome). $(D)$ Freestanding lipid bilayer. (E) Single and double lipid bilayers on solid supports. 
O.G. Mouritsen

techniques, such as atomic-force microscopy, provided the monolayer is transferred to a solid support. The combined information obtained will hence cover scales ranging from the molecular scale, over the nanometer scale, to the micron and macroscopic length scales.

Importantly, lipid monolayers on air-water interfaces are good models to probe, in a welldefined setting, the interactions between the lipid compartment of cell membranes and various agents that are active on membranes, such as solutes, drugs, enzymes, and water-soluble and amphiphatic peptides.

An example of lateral monolayer lateral structure imaged by multiphoton excitation fluorescence microscopy using laurdan is shown in Figure 2A. The image illustrates the coexistence between liquid-expanded and liquid-condensed phases in a DPPC (dipalmitoylphosphatidylcholine) monolayer at an air-water interface (Brewer et al. 2010).

\section{SUPPORTED LIPID BILAYERS AND MULTILAYERS}

Freestanding lipid bilayers (cf. Fig. 1D) can be transferred to solid supports under full aqueous conditions (cf. Fig. 1E and Fig. 2B-F) using, for example, Langmuir-Blodgett deposition techniques, or self-assembled by using rehydration subsequent to spin-coating techniques, or by explosion of a vesicular dispersion onto an appropriate hydrophilic surface. Using proteoliposomes it is also possible to produce supported lipid bilayers with integral membrane proteins incorporated (cf. Fig. 2D). The correct incorporation and folding of large integral membrane proteins can be facilitated using solid supports with appropriate soft cushions in the form of grafted polymer layers or brushes (Tanaka and Sackmann 2005).

Proximal lipid bilayers on solid supports are significantly influenced by interactions with the substrate, and their thermodynamics and lateral organization and order is affected by the support (Jensen et al. 2007; Keller et al. 2005). Hence, caution should be exerted when comparing data for supported bilayers with corresponding data for free-standing bilayers. The influence of the support can be significantly suppressed by adding a second, distal bilayer on top of the proximal one (cf. Fig. 2E) (e.g., using spin-coating techniques [Simonsen and Bagatolli 2004]).

The advantage of supported bilayers is that they can be manipulated and imaged in advanced fluorescence and atomic-force microscopes, hence revealing their lateral structure on scales from nanometers to microns (cf. Fig. 2B-E). Under suitable conditions, reconstituted proteins can also be visualized as shown in Figure 2D. In supported membranes with high protein contents (e.g., in natural membranes of the purple membranes of Halobacterium halobium), the crystalline arrays of bacteriorhodopsin reveal details of the protein arrangements (Oesterhelt et al. 2000; Butt 2009).

The top layer of double-supported lipid bilayers can help answer questions regarding lateral membrane structure on scales below the diffraction limit, and atomic-force microscopy can lead to valuable real-space information on membrane domains, phase separation, and molecular organization of model membranes (Leidy et al. 2002). As an example, the image in Figure 2C shows the small-scale ripple structure of the top layer of a DLDP/DPPC doublesupported bilayer. In some cases dynamical phenomena can be studied-for example, the action of a phospholipase-assisted hydrolysis of a lipid bilayer as illustrated in Figure $2 \mathrm{~F}$ (Simonsen 2008).

Studies of supported bilayers may eventually shed some light on one of the major outstanding and elusive questions in membrane biology, that of lipid diversity. It remains a puzzle why biological membranes consist of hundreds of different kinds of lipids. Part of the answer is likely to lie in the dynamics of the lateral structure of the lipid-bilayer component of the membranes.

\section{CLOSED BILAYERS: VESICLES, LIPOSOMES, AND THE SUVs, THE LUVs, AND THE GUVs}

Vesicles and unilamellar liposomes are possibly the simplest models of closed cell membranes (cf. Fig. 1C). They are freestanding in water, 

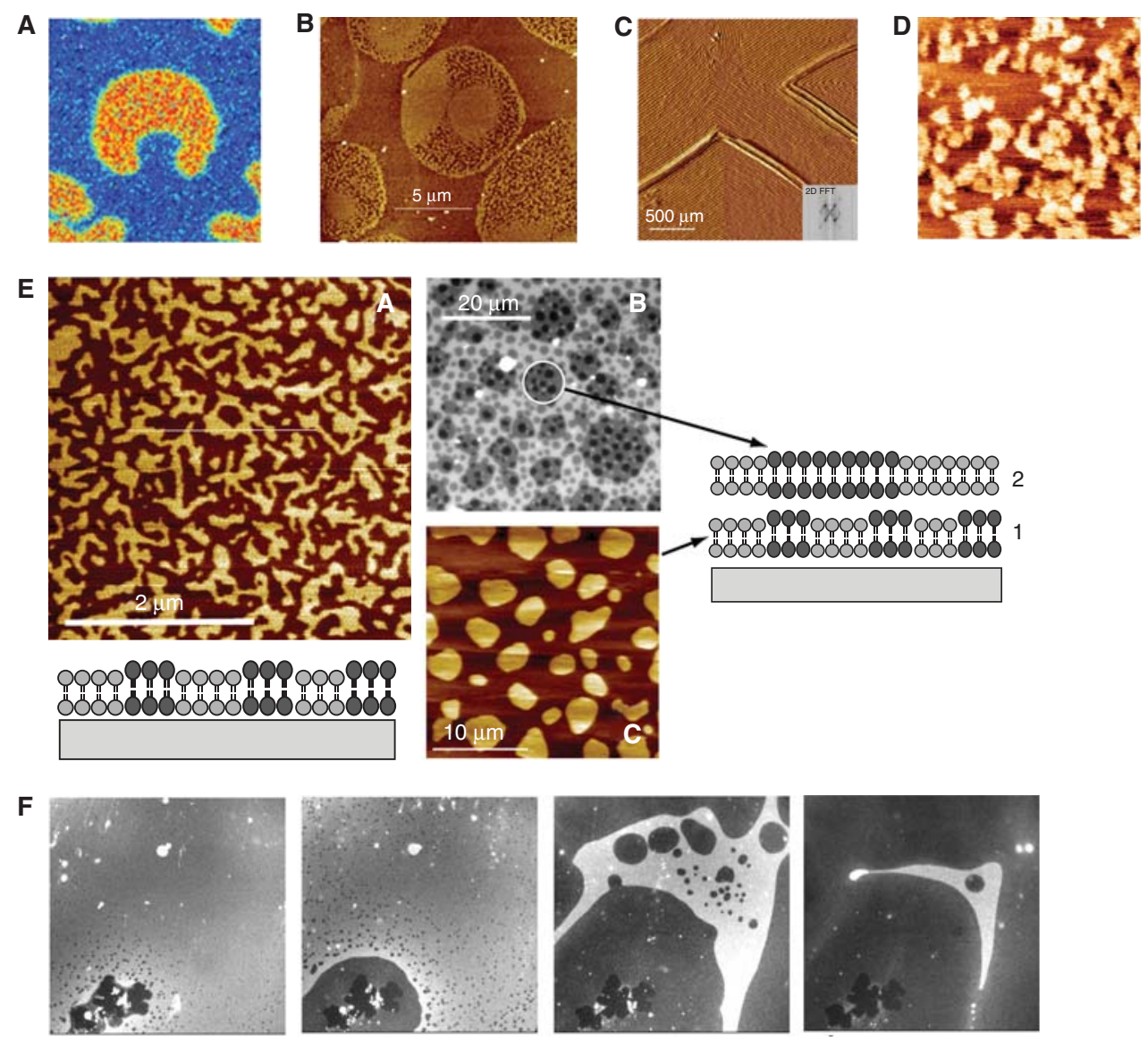

Figure 2. Lateral structure of lipid mono- and bilayers. (A) Multiphoton excitation fluorescence microscopy image, using laurdan, of the lateral structure of a DPPC monolayer in the liquid-extended liquid-condensed coexistence region at $8.8 \mathrm{mN} / \mathrm{m}$ and $21^{\circ} \mathrm{C}$. (Adapted from Brewer et al. 2010; reprinted with permission from Elsevier (C) 2010). (B) Atomic-force microscopy image of the lateral structure of a single-supported bilayer of native pulmonary surfactant. (Adapted from Bernardino de la Serna et al. 2004.) (C) Atomic-force microscopy image of the lateral structure of the top bilayer of a double-supported bilayer system of DLPC/DPPC 1:1 at $20^{\circ} \mathrm{C}$ revealing the occurrence of ripple structures with a periodicity of $30 \mathrm{~nm}$. (Courtesy of Dr. Uffe Bernchou Jensen.) (D) Lateral structure of a supported lipid bilayer reconstituted with individually discernable aquaporins. The image is $100 \mathrm{~nm} \times 100 \mathrm{~nm}$. (Courtesy of Dr. Danielle Keller.) (E) Atomic-force (E:A) and fluorescence microscopy $(E: B$ and $E: C)$ images of the lateral structure of single- and double-supported lipid bilayer of the raft mixture DOPC/DPPC/Chol 2:2:1 spin coated onto a mica surface. (Adapted from Jensen et al. 2007; reprinted courtesy of Dr. Adam Cohen Simonsen.) (F) Fluorescence microscopy images $(120 \mu \mathrm{m} \times 120 \mu \mathrm{m})$ of the time evolution of the lateral structure of a single-supported POPC lipid bilayer being hydrolyzed by secretory phospholipase $\mathrm{A}_{2}$. (Adapted from Bernchou Jensen and Simonsen 2005; reprinted with permission from Elsevier (C) 2005.)

they are single-walled, they are closed onto themselves, and they hence separate an aqueous compartment from the environment. As membrane models, the vesicles have the advantage that they can be formed in different sizes, SUVs (small unilamellar vesicles, $<100 \mathrm{~nm}$ in diameter), LUVs (large unilamellar vesicles, $<1000 \mathrm{~nm}$ in diameter), and GUVs (giant unilamellar vesicles, $>1000 \mathrm{~nm}$ in diameter). Hence, it is possible to study effects of membrane permeability barriers and curvature. Dispersions of vesicles give access to measurement 
O.G. Mouritsen

of ensemble properties (e.g., thermodynamic and structural properties).

GUVs are particularly useful membrane models because they are large enough to be studied individually in a visual microscope (Bagatolli 2006, 2010). Moreover they can be manipulated by micromechanical devices and their mechanical properties can be assessed (e.g., by pipette aspiration techniques [Evans and Skalak 1980]). In recent years, GUVs have established themselves as a key model and versatile laboratory for answering a plethora of membrane questions, not least in relation to domain formation. It is noteworthy that special fluorescent lipid analogs such as laurdan and prodan can be used as simultaneous spectroscopic probes that not only reveal lateral structure but also aspects of the lipid chain order. This is of particular importance for questions regarding the order of domains related to membrane rafts (Dietrich et al. 2001; Fidorra et al. 2009).

Figure 3 shows some examples of the type of information that advanced fluorescence microscopy can reveal on lateral membrane structure in GUVs formed by simple and more complex lipid mixtures.

A quantitative analysis of images as those shown in Figure 3 can answer questions regarding phase diagrams and phase equilibria and domain size distributions, as well as domain shapes and their evolution in time (Baumgart et al. 2003). Moreover, modifications in the lateral organization can be studied under the influence of membrane-active agents, drugs, and enzymes.

\section{FOUR CONSPICUOUS FEATURES OF LIPID MEMBRANES REVEALED BY MEMBRANE MODELS}

Studies of membrane models as described above have provided critical answers regarding several fundamental questions pertaining to biomembranes. We shall briefly mention four of the more important lessons from such studies. The results are obtained by combining insight from using a range of experimental techniques, some of them mentioned above,

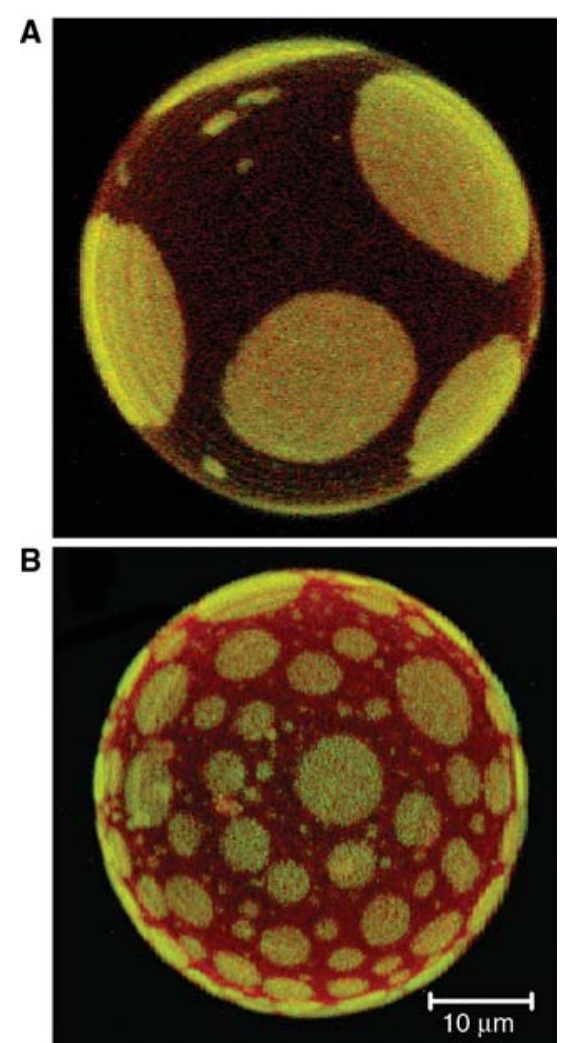

Figure 3. Lateral structure in giant unilamellar vesicles (GUVs) as visualized by fluorescence microscopy. (A) Mixture of DOPC/DPPC/Chol 2:2:1 at $20^{\circ} \mathrm{C}$. (B) Native pulmonary surfactant mixtures. (B, Adapted from Bernardino de la Serna et al. 2004; reprinted with permission from The Journal of Biological Chemistry (C) 2004.)

in combination with theoretical modeling and computer simulation (Mouritsen 2005).

\section{The Lateral Pressure Profile}

The transverse structure of a lipid bilayer is possibly the most overlooked, but at the same time most obvious and fundamental property of a membrane (Cantor 1997a). Its transbilayer shape, as illustrated in Figure 4A, is highly nontrivial, revealing regions of expansive (positive) pressures and regions of large tensile (negative) pressures. The pressure profile owes its existence to the two opposed oil-water interfaces 

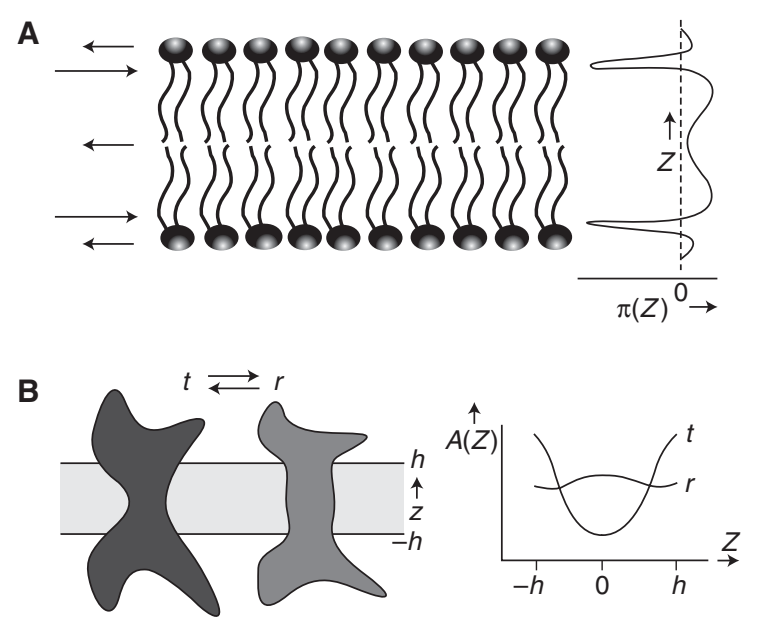

Figure 4. Transbilayer structure of lipid bilayers. (A) Schematic illustration of the lateral pressure profile, $\pi(z)$ of a lipid bilayer, revealing regions of expansive ( positive) pressures and regions of large tensile (negative) pressures. $(B)$ Illustration of how changes in the lateral pressure profile may shift the equilibrium between two conformational states $(t, r)$ of an integral membrane protein. $A(z)$ describes how the protein cross-sectional area varies across the bilayer from $-h$ to $h$. ( $B$, Adapted from Cantor 1997a; reprinted with permission from The American Chemical Society (C) 1997.)

sandwiching the hydrophobic core of the membrane interior.

The large variations in pressure, ranging from the equivalent of a hundred to a thousand atmospheres, are a direct consequence of the distribution of the oil-water interfacial tension over a bilayer thickness of $\sim 5 \mathrm{~nm}$.

The lateral pressure profile puts distinct constraints on the interaction between the membrane and integral proteins, and it is believed that protein and channel function may be regulated by the lipids via alterations in the pressure profile. In particular it has been surmised that certain solutes, ligands, and drugs exert part of their action on membrane proteins and receptors via changes in the pressure profile as illustrated schematically in Figure 4B. A prominent example is that of the molecular mechanism of general anesthesia, which is likely to involve modulation of the pressure profile, and then in turn the lipid-protein interactions, by the localization of the potent anesthetic agents in the interfacial regions of the membrane surface (Cantor 1997b).

The pressure profile, and its dependence on bilayer composition and thermodynamic conditions, has been carefully worked out by theoretical models and computational techniques (Patra 2005; Sonne et al. 2005). Still no experimental technique has been devised to actually measure the profile, the reason being that no probe has been found that faithfully and without perturbing the system can measure pressure differences on the subnanometer scale.

\section{Cholesterol and the Liquid-Ordered Phase}

A single, special kind of lipid, cholesterol, has come to play a key role in the elucidation of membrane organization and domain formation (Mouritsen and Zuckermann 2004; Mouritsen 2010). Being the single most abundant type of lipid in all plasma membrane, typically in a molar concentration of $\sim 30 \%$, a long-standing membrane question has been how cholesterol regulates membrane structure and function.

By the proposal of a new type of membrane phase, the liquid-ordered phase (Ipsen et al. 1987), that results as a compromise between cholesterol's schizophrenic affinity for fluid and solid lipid phases, cholesterol was shown to control membrane organization on different length scales. The proposal was based on a simple theoretical model of lipid bilayers and it was 
inspired by experimental NMR (nuclear magnetic resonance) and calorimetry data for the cholesterol-DPPC system (Vist and Davis 1990). The liquid-ordered phase was shown to be a fundamental physical and thermodynamic consequence of the symmetry of the binary lipid-cholesterol mixture, with two liquid phases of the same fluid symmetry but a different degree of acyl-chain order: the liquiddisordered (fluid) phase and the liquid-ordered phase. The resulting phase diagram is a manifestation of the basic facts that cholesterol has a preference for ordered acyl lipid chains (i.e., chain states characteristic of the solidordered [gel] phase), but at the same time it is more soluble in the fluid phase. This apparent conflict is resolved by inducing an intermediate phase-that is, a phase that is ordered from the point of view of the conformational structure of the lipid chains but is disordered from the point of view of the lateral positions of the molecules.

The proposal of the liquid-ordered phase from a simple membrane model was hence indirectly responsible for consolidating a sound physical basis for the functional membrane platforms that underlie the phenomenological raft hypothesis (Simons and Ikonen 1997).

Even if the liquid-ordered phase was proposed to exist in binary lipid-cholesterol systems, only little conclusive evidence is available as yet of the presence of a macroscopic liquid-ordered phase and macroscopic phase coexistence between liquid-ordered-liquiddisordered phases in binary lipid bilayers. Nevertheless, there is now a large body of experimental work that directly or indirectly has shown unequivocally the existence of smaller microscopic domains in model membranes, possibly of the size of tens of nanometers (Veatch et al. 2004; Pencer et al 2007; Davis et al. 2009).

Lateral Membrane Structure: Phases, Domains, and Rafts

Both theoretical and experimental studies of model membranes, monolayers as well as bilayers, very early showed that membranes are structured laterally, first of all as a consequence of the many-bodyness and cooperative behavior of the system (Mouritsen and Jørgensen 1994; Mouritsen 2005). Phase equilibria can lead to large-scale phase separation, and local fluctuations in composition and density can lead to small-scale lipid domain formation. Inhomogeneities in the form of defects and coupling to cytoskeleton and external structures may eventually control the length scale of the domains. Examples of lateral organization in some simple model membranes were shown in Figures 2 and 3.

Subsequent to the proposal of the raft hypothesis, a large number of model studies have been performed on special lipid mixtures that are believed to be raft formers. The typical system is a ternary mixture including cholesterol together with a lipid with a high melting point (e.g., a saturated lipid or sphingolipid) and one with a low melting point (e.g., a monounsaturated or polyunsaturated lipid). Many ternary phase diagrams have been worked out (Veatch and Keller 2003; Jensen et al. 2007; Goñi et al. 2008), and paradoxically the identification of the liquid-ordered phase in coexistence with a fluid lipid phase has been much more easy in ternary systems compared to binary lipid-cholesterol systems (Bagatolli et al 2010; Mouritsen 2010).

\section{Membrane Mechanics}

Because of its very small thickness compared to its extension, a lipid bilayer may be modeled as a two-dimensional, flexible sheet imbedded in a three-dimensional space (Evans and Skalak 1980). Because membranes usually are prepared in a tensionless state and the surface tension hence is negligible, the energetics of the sheet is controlled by the mechanical modules (i.e., the bending modulus, $\kappa$, and the Gaussian curvature modulus, $\kappa_{\mathrm{G}}$ ) and described by the Helfrich expression

$$
d E_{\text {surface }}=\left[\frac{\kappa}{2}\left(\frac{1}{R_{1}}+\frac{1}{R_{2}}-\frac{2}{R_{0}}\right)^{2}+\frac{\kappa_{\mathrm{G}}}{R_{1} R_{2}}\right] d A,
$$


in which $d E_{\text {surface }}$ is the energy differential associated with an area deformation $d A . R_{1}$ and $R_{2}$ are the principal radii of curvature of the sheet, and $R_{0}$ is a measure of the spontaneous curvature. Because the membrane is assumed to be fluid, there is no term in Equation (1) accounting for resistance to shearing. For closed bilayers like vesicles, the Gaussian term integrates to a topological constant when the total energy is evaluated by integrating Equation (1) over the entire surface of the vesicle. Hence, only the bending term and the bending modulus $\kappa$ contribute to the mechanics in this approximation. Equation (1) would have to be modified if area differences between the two monolayers are significant (Miao et al. 1994).

The Helfrich Hamiltonian in Equation (1) has turned out to be a remarkably good model of membrane mechanics and it has been successful in accounting for the various shapes and shape transformations of lipid vesicles and even cells like the erythrocyte (Lim et al. 2002). The model has also proven to be a workhorse in the laboratory for determining mechanical parameters of lipid membranes, using micromechanical measurements such as vesicle fluctuation analysis, in which the undulations of fluctuating GUVs in the microscopy via Fourier analysis (Henriksen et al. 2004) can yield the bending modulus, even under physiological conditions (Méléard et al. 2009). In particular, this type of model approach has answered important questions regarding the effect of antimicrobial, amphiphatic peptides or sterols on membrane mechanics, thereby supplementing parallel model studies of the same systems using more conventional thermodynamic and spectroscopic investigations.

\section{RECONSTITUTED MEMBRANES AND LIPID-PROTEIN INTERACTIONS}

Modeling of biological membranes typically progresses in steps, starting with simple lipid bilayers with one or two lipid species, then proceeds to more complex lipid mixtures, including special lipids such as sterols, sphingolipids, and glycolipids. Another step may be using the full lipid extract from cell membranes.
This type of modeling focuses on the lipid properties and it has provided the foundation for interpretation of many experiments on real biological membranes and it has revealed to which extent lipids marshal membrane structure and function (Mouritsen 2005).

Parallel to this line of modeling is the formulation of models attempting to capture the essentials of protein-membrane interactions (e.g., integral membrane proteins). Most of the proposed models, however, fall short of describing real membranes in which half of the mass is proteins. Experimental models are typically lipid-protein recombinants in which purified membrane proteins are reconstituted into vesicles and liposomes of a well-defined lipid composition (de Kruiff 2004; Tamm 2005; Perez-Gil 2008).

Important membrane questions that the models should answer are related to, for example, lateral distribution and organization of the proteins in the plane of the membrane, the effect of lipid-protein interactions on lipid domains and rafts, the structure of the lipids in close proximity to the proteins, the possible impact of lipid properties on protein structure and hence function, and the possibly lipid-mediated interactions between proteins. These questions are all in some way or another interrelated.

To illustrate the potential of simple theoretical models for answering some of these systems we shall briefly describe how the concepts of hydrophobic matching, lateral pressure profile, and lipid propensity for curvature have proven to be useful.

Hydrophobic matching between the hydrophobic core of lipid bilayers and the hydrophobic stretch of integral membrane proteins has been considered by several authors (Sackmann 1984; Mouritsen and Bloom 1984; Jensen and Mouritsen 2004; Andersen and Koeppe 2007) as a key determinant of lipid protein interactions (cf. Fig. 5A). The idea is that lack of a perfect match carries an energy penalty that basically amounts to the elastic distortion of the lipid matrix around the protein. For a sufficient large value of this penalty, the protein may yield and undergo a conformational change, hence offering a mechanism for lipid-mediated 
O.G. Mouritsen
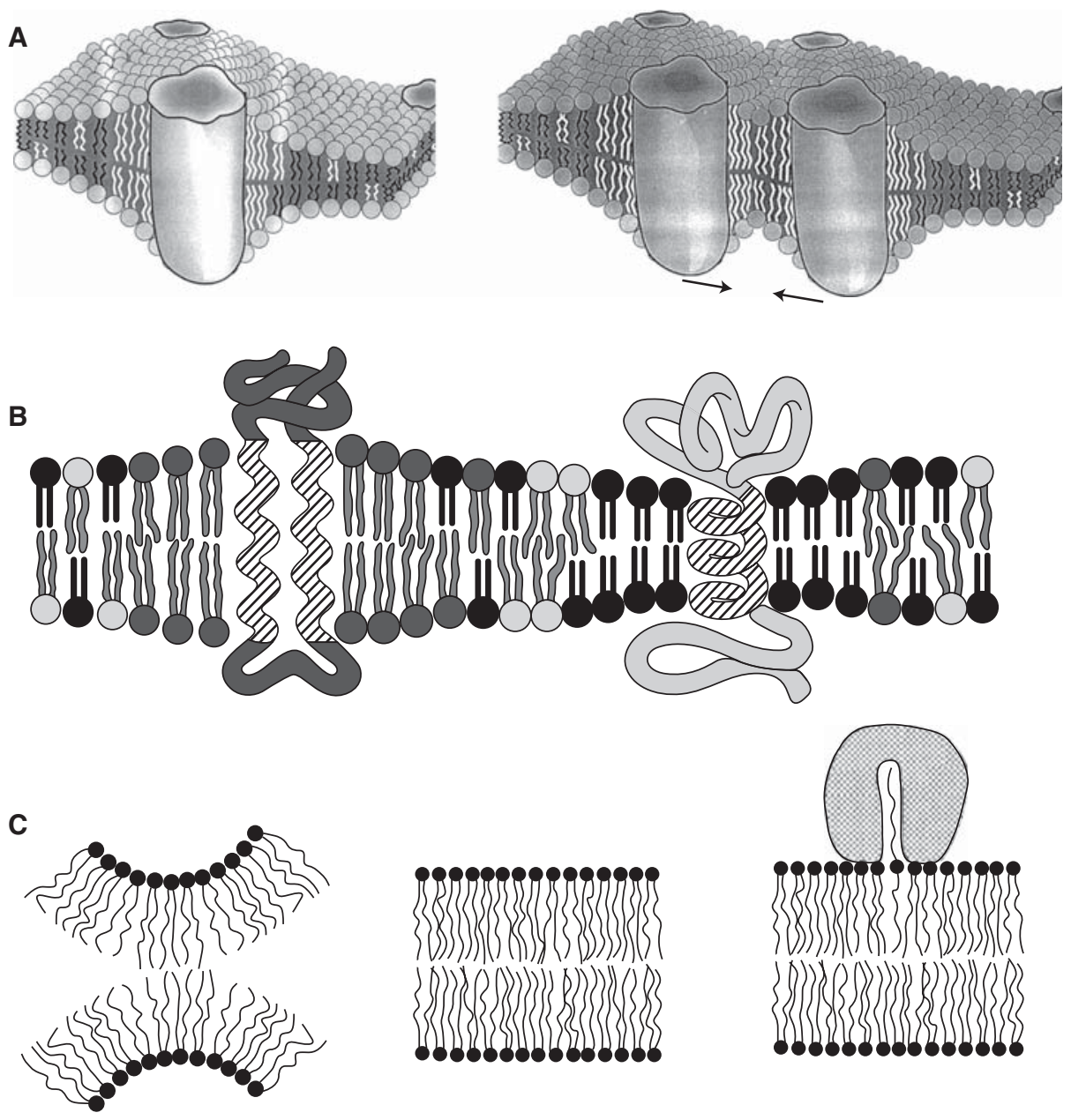

D

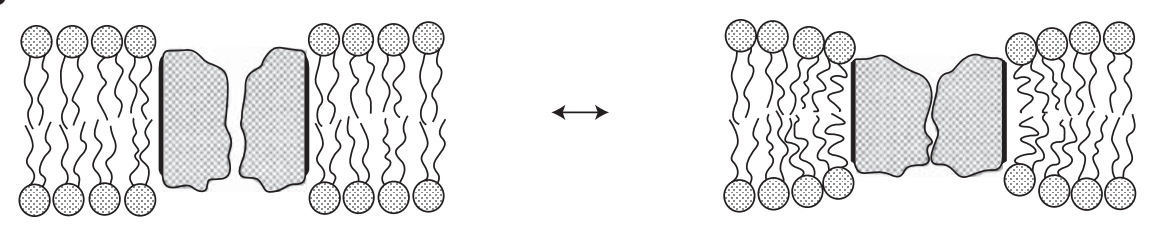

Figure 5. Lipid-protein interactions in membranes. (A) Schematic illustration of the principle of hydrophobic matching between lipid bilayers and integral membrane proteins. In the case of a mismatch, the deformation in the lipid matrix may induce an indirect, lipid-mediated attraction between the proteins. $(B)$ Schematic illustration of a conformational change in an integral membrane protein induced by changes in the hydrophobic mismatch condition. (Data from Sackmann 1984.) (C) Release of the curvature stress in a lipid bilayer, composed of two lipid monolayers with spontaneous curvature, via the formation of the extended lipid chain conformation. One of the tails of the lipid molecule is captured in a hydrophobic pocket of a peripheral membrane protein. (Courtesy of Dr. P.K.J. Kinnunen.) (D) Curvature stress may induce conformational changes in a membrane channel and hence shift the equilibrium between an open and a closed state. (Courtesy of Dr. O.S. Andersen.) 
effects on protein function, as illustrated in Figure 5B.

Another consequence of hydrophobic matching is the possibility of sorting, selection, or enrichment of certain lipids near the protein (Dumas et al. 1997, 1999). This is a purely physical effect and does not require special binding affinity of certain lipids for proteins. Obviously, specific interactions (e.g., by electrostatics) will add to this picture and may in some case overrule the matching.

For larger protein concentrations, the hydrophobic matching provides a mechanism for lipid-mediated protein-protein interactions, typically attractive as illustrated in Figure 5A, which will provide a driving force for protein aggregation and possibly crystallization in the plane of the membrane. The range of this protein-protein interaction will be controlled by the coherence length of the correlations between the lipids. These correlations can get rather long-ranged, in particular near special (critical) points in the system phase diagram. This effect is a version of the more general wetting phenomenon (Gil et al. 1997, 1998). Although a strictly equilibrium phenomenon, critical points in complex lipid mixtures (e.g., those containing cholesterol) may hence be interesting to consider in relation to membrane organization (Gil et al. 1997; Veatch et al. 2007; Honerkamp-Smith et al. 2008; Bagatolli et al. 2010). This is another example of the power of membrane models to provide intuition regarding important membrane questions.

The hydrophobic matching principle has been important in substantiating the concept of membrane rafts (Lingwood and Simons 2010) which are domains enriched in cholesterol and high-melting lipids and therefore generally thicker than the membrane matrix in which they reside. Matching then furnishes a mechanism for protein selection where those proteins that match the raft thickness the better (e.g., via appropriate acylation or prenylation) are recruited to the raft. Conversely, they can be released from the raft by appropriate enzymatic modification of the proteins. Hence, the concept of hydrophobic matching helps to establish a semiquantitative physical framework for signaling cascades.
It should be emphasized that in the present picture, lipid domains and rafts are dynamically maintained entities (Mayor and Rao 2004)that is, they are by no means static but highly fluctuating and dynamic. They owe their existence to the correlations and many-bodyness of the of the entire membrane assembly.

The propensity of some lipids for inducing curvature stress and possibly nonlamellar phases provides, via the lateral pressure profile, provides another mechanism for lipid-protein interactions (Marsh 2007) as illustrated in Figure $4 \mathrm{~B}$. This mechanism is not necessarily independent from the hydrophobic matching mechanism. An illustration is given in Figure 5, $\mathrm{C}$ and $\mathrm{D}$, in the case of release of curvature stress by binding a peripheral membrane protein and a shift between open and closed conformations of a membrane channel, respectively. Examples of systems that have been shown to operate according to these principles are the binding of cytochrome $c$ to a membrane surface (Touminen et al. 2002) and the opening and closing of gramicidin A channels (Andersen and Koeppe 2007), respectively.

\section{A CALL FOR NEW MEMBRANE MODELS}

Virtually all existing theoretical models of membranes are based on equilibrium considerations. The same is true of most experimental models that typically are performed as test tube experiments. Many membrane systems studied in the laboratory are not considered in the full functional state and the experimental measurements usually pertain to some kind of equilibrium or near-equilibrium state. Obviously, real biological membranes are far from equilibrium or at best in a driven steady-state situation. In any case, the well-known principles from equilibrium thermodynamics do not apply. A typical example is a membrane with an ion pump that is driven by some kind of energy transduction mechanism (Sabra and Mouritsen 1998; Girard et al. 2005). Another example is the binding of ligands to receptors in which the binding is influenced by a force (e.g., the binding of collectins in the innate immune system to sugar groups on invading 
O.G. Mouritsen

pathogens [Thormann et al. 2007]). A third example is the set of responsive membranes in the dermal barrier that is subject to a gradient in water chemical potential (Sparr and Wennerström 2001). A fourth example is the morphogenesis of the endoplasmatic reticulum and the Golgi apparatus with membrane that owe their existence to nonequilibrium conditions of flow of energy and matter (Kühnle et al. 2010). It is interesting in this context to note that the plasma membrane can undergo phase separation when the cell dies (Lingwood and Simons 2010).

We are only about to begin facing questions as to how we by models study membrane structure in the functional state. For instance, how does bilayer transverse and lateral order become modified in an active membrane subject to transport, signaling, and enzymatic processes, and how do these processes in turn become controlled by the nonequilibrium state of the lipid matrix? Only few clues exist (Sabra and Mouritsen 1998; Høyrup et al. 2002; Girard et al. 2005; Turner et al. 2005; Lomholt 2006; Fan et al. 2010) and progress is hampered by the lack of appropriate, quantitative model systems that can be analyzed with the accuracy we are used to from equilibrium conditions.

Hence there is an urgent call for new models, experimental as well as theoretical, that can establish a framework for future work for answering pressing questions regarding membrane structure and function in nonequilibrium states.

\section{MODEL MEMBRANES FOR HEALTH AND TECHNOLOGY}

Membrane models have in many cases served as stepping stones in translating the insight from lipid membrane biophysics and biochemistry into technological applications, it be design of novel functional materials, nano-scale encapsulation technologies for foodstuff and drugs, sensors, and living technology.

As specific examples can be mentioned the design of two concrete new designs of liposomebased drug delivery systems for cancer therapy whose existence is to a large extent based on extensive physical modeling. The first one is a thermosensitive liposome for delivering of anticancer drugs in which the essential triggering mechanism is a lipid bilayer phase transition that is tuned to take place a few degrees above physiological temperatures (Ponce et al. 2006). By heating tumors, in which the liposomes accumulate, from the outside the liposomal membranes go through their phase transition and become leaky. As a consequence, the encapsulated drug leaks out of the liposomes exactly where it is needed. The other example is a liposome that is made sensitive to the action of an endogenously up-regulated secretory phospholipase $\mathrm{A}_{2}$ in cancer tissue. On accumulation in the tumor of intravenously administered liposomes with encapsulated anticancer drugs, the phospholipase opens the liposomal carrier and the drugs leak out (Jørgensen et al. 2002; Andresen et al. 2005). This latter system can furthermore be expanded to include lipid prodrugs or double prodrugs that are turned into drugs by the action of the phospholipase, precisely at the target (Pedersen et al. 2010).

Both mentioned liposomal drug delivery systems have already been taken to clinical trials. They are good examples of how membrane models may provide answers to important problems in health and biotechnology.

\section{ACKNOWLEDGMENTS}

Illuminating discussion with members of MEMPHYS is gratefully acknowledged. MEMPHYS-Center for Biomembrane Physics is supported by the Danish National Research Foundation.

\section{REFERENCES}

Andersen OS, Koeppe REII. 2007. Bilayer thickness and membrane protein function: An energetic perspective. Annu Rev Biophys Biomol Struct 36: 107-130.

Andresen TL, Jensen SS, Jørgensen K. 2005. Advanced strategies in liposomal cancer therapy: Problems and prospects of active and tumor specific drug release. Prog Lip Res 44: 68-97.

Bagatolli LA. 2006. To see or not to see: Lateral organization of biological membranes and fluorescence microscopy. Biochim Biophys Acta 1758: 1541-1556. 
Bagatolli LA, ed. 2010. BBA special issue: Microscopy imaging of membrane domains. Biochim Biophys Acta 1798: $1285-1456$.

Bagatolli LA, Ipsen JH, Simonsen AC, Mouritsen OG. 2010. An outlook on organization of lipids in membranes: Searching for a realistic connection with the organization of biological membranes. Prog Lip Res 49: 378-389.

Bangham AD, Horne RW. 1964. Negative staining of phospholipids and their structural modification by surfaceactive agents as observed in the electron microscope. J Mol Biol 8: 660-668.

Baumgart T, Hess ST, Webb WW. 2003. Imaging coexisting fluid domains in biomembrane models coupling curvature and line tension. Nature 425: 821-824.

Bergelson L, ed. 1995. Domain organization in biological membranes. Mol Memb Biol 12: 1-162.

Bernardino de la Serna J, Perez-Gil J, Simonsen AC, Bagatolli LA. 2004. Cholesterol rules: Direct observation of the coexistence of two fluid phases in native pulmonary surfactant membranes at physiological temperatures. J Biol Chem 279: 40715-40722.

Bernchou Jensen U, Simonsen AC. 2005. Shape relaxations in a fluid supported membrane during hydrolysis by phospholipase A2. Biochim Biophys Acta 1715: 1-5.

Brewer J, Bernardino de la Serna J, Wagner K, Bagatolli LA. 2010. Multiphoton excitation fluorescence microscopy in planar membrane systems. Biochim Biophys Acta 1798: 1301-1308.

Butt H. 2009. Imaging the membrane protein bacteriorhodopsin with the atomic force microscope. Biophys J $\mathbf{5 8}$ 1473-1480.

Cantor RS. 1997a. Lateral pressures in cell membranes: A mechanism for modulation of protein function. J Phys Chem 101: 1723-1725.

Cantor RS. 1997b. The lateral pressure profile in membranes: A physical mechanism of general anesthesia. Biochemistry 36: 2339-2344.

Cullis PR, de Kruijff B. 1979. Lipid polymorphism and the functional roles of lipids in biological membranes. Biochim Biophys Acta 559: 399-420.

Davis JH, Clair JJ, Juhasz J. 2009. Phase equilibria in DOPC/ DPPC- $\mathrm{d}_{62} /$ cholesterol mixtures. Biophys J 96: 521-539.

de Kruiff B. 2004. BBA special issue: Lipid-protein interactions. Biochim Biophys Acta 1666: 1-288.

Dietrich D, Bagatolli LA, Volovyk Z, Thompson NL, Levi M, Jacobson K, Gratton E. 2001. Lipid rafts reconstituted in model membrane. Biophys J 80: 1417-1428.

Dumas F, Lebrun MC, Tocanne J-F. 1999. Is the protein/ lipid hydrophobic matching principle relevant to membrane organization and functions? FEBS Lett 458: 271-277.

Dumas F, Sperotto MM, Lebrun MC, Tocanne JF, Mouritsen OG. 1997. Molecular sorting of lipids by bacteriorhodopsin in dilauroylphosphatidylcholine/distearoylphosphatidylcholine lipid bilayers. Biophys J 73: 1940-1953.

Dynarowicz-Latka P, Dhanabalan A, Oliveira ON Jr. 2001. Modern physicochemical research on Langmuir monolayers. Adv Colloid Interface Sci 91: 221-293.

Engelman DM. 2005. Membranes are more mosaic than fluid. Nature 438: 578-580.
Evans EA, Skalak R. 1980. Mechanics and thermodynamics of biomembranes. CRC Press, Boca Raton, FL.

Fan J, Sammalkorpi M, Haataja M. 2010. Influence of nonequilibrium lipid transport, membrane compartmentalization, and membrane proteins on the lateral organization of the plasma membrane. Phys Rev E 81: 011908.

Fidorra M, Garcia A, Ipsen JH, Härtel S, Bagatolli LA. 2009. Lipid domains in giant unilamellar vesicles and their correspondence with equilibrium thermodynamic phases: A quantitative fluorescence microscopy imaging approach. Biochim Biophys Acta 1788: 2142-2149.

Gil T, Ipsen JH, Mouritsen OG, Sabra MC, Sperotto MM, Zuckermann MJ. 1998. Theoretical analysis of protein organization in lipid membranes. Biochim Biophys Acta 1376: $245-266$.

Gil T, Sabra MC, Ipsen JH, Mouritsen OG. 1997. Wetting and capillary condensation as means of protein organization in membranes. Biophys J 73: 1728-1741.

Girard P, Prost J, Bassereau P. 2005. Passive or active fluctuations in membranes containing proteins. Phys Rev Lett 94: 088102.

Goñi FM, Alonso A, Bagatolli LA, Brown RE, Marsh D, Prieto M. 2008. Phase diagrams of lipid mixtures relevant to the study of membrane rafts. Biochim Biophys Acta 1781: 665-684.

Gorter EF, Grendel F. 1925. On biomolecular layers of lipoids on chromacytes of blood. J Exp Medicine 41: 439-443.

Heimburg T. 2007. Thermal biophysics of membranes. Wiley-VCH, Berlin.

Heimburg T, ed. 2009. Themed issue: Membrane biophysics. Soft Mat 5: 3129-3364.

Henriksen J, Rowat AC, Ipsen JH. 2004. Vesicle fluctuation analysis of the effects of sterols on membrane bending rigidity. Eur Biophys J 33: 732-741.

Honerkamp-Smith AR, Cicuta P, Collins MD, Veatch SL, den Nijs M, Schick M. 2008. Line tensions, correlation lengths, and critical exponents in lipid membranes near critical points. Biophys J 95: 236-246.

Høyrup P, Jørgensen K, Mouritsen OG. 2002. Phospholipase A2-An enzyme that is sensitive to the physics of its substrate. Europhys Lett 57: 464-470.

Ipsen JH, Karlström G, Mouritsen OG, Wennerström H, Zuckermann MJ. 1987. Phase equilibria in the phosphatidylcholine-cholesterol system. Biochim Biophys Acta 905: 162-172.

Israelachvili JN. 1977. Refinement of the fluid-mosaic model of membrane structure. Biochim Biophys Acta 469: $221-225$.

Israelachvili JN. 1992. Intermolecular and surface forces, 2nd ed. Academic Press, New York.

Jacobson K, Mouritsen OG, Anderson GW. 2007. Lipid rafts: At a crossroad between cell biology and physics. Nature Cell Biol 9: 7-14.

Jensen MØ, Mouritsen OG. 2004. Lipids do influence protein function-The hydrophobic matching hypothesis revisited. Biochim Biophys Acta 1666: 205-226.

Jensen MH, Morris EJ, Simonsen AC. 2007. Domain shapes, coarsening, and random patterns in ternary membranes. Langmuir 23: 8135-8141. 
O.G. Mouritsen

Jørgensen K, Davidsen J, Mouritsen OG. 2002. Biophysical mechanisms of phospholipase A2 activation and their use in liposome-based drug delivery. FEBS Lett 531: 23-27.

Keller D, Larsen NB, Møller IM, Mouritsen OG. 2005. Decoupled phase transitions and grain-boundary melting in supported phospholipid bilayers. Phys Rev Lett 94: 025701.

Kusumi A, Suzuki K. 2005. Toward understanding the dynamics of membrane-raft-based molecular interactions. Biochim Biophys Acta 1746: 234-251.

Kühnle J, Shillcock J, Mouritsen OG, Weiss M. 2010. A modeling approach to the self-assembly of the Golgi apparatus. Biophys J 98: 2839-2847.

Leidy C, Kaasgaard T, Crowe JH, Mouritsen OG, Jørgensen K. 2002. Ripples and the formation of anisotropic lipid domains: Imaging two-component supported double bilayers by atomic force microscopy. Biophys $J$ 83: $2625-2633$.

Lim HW G, Wortis M, Mukhopadhyay R. 2002. Stomatocyte-discocyte-echinocyte sequence of the human red blood cell: Evidence for the bilayer-couple hypothesis from membrane mechanics. Proc Natl Acad Sci 99: 16766-16769.

Lingwood D, Simons K. 2010. Lipid rafts as a membraneorganizing principle. Science 327: 46-50.

Lomholt M. 2006. Fluctuation spectrum of quasispherical membranes with force-dipole activity. Phys Rev E 73: 061914.

Luzzati V, Tardieu A, Gulik-Krzywicki T. 1968. Polymorphism of lipids. Nature 217: 1028-1030.

Marčelja S. 1976. Lipid-mediated protein interaction in membranes. Biochim Biophys Acta 455: 1-7.

Marrink SJ, de Vriesa AH, Tieleman DP. 2009. Lipids on the move: Simulations of membrane pores, domains, stalks and curves. Biochim Biophys Acta 1788: 149-168.

Marsh D. 2007. Lateral pressure profile, spontaneous curvature frustration, and the incorporation and conformation of proteins in membranes. Biophys J 93: 3884-3899.

Mayor S, Rao M. 2004. Rafts: Scale-dependent, active lipid organization at the cell surface. Traffic 5: 231-240.

Méléard P, Bagatolli LA, Pott T. 2009. Giant unilamellar vesicle electroformation from lipid mixtures to native membranes under physiological conditions. Methods Enzymol 465: 161-176.

Merz KM Jr, Roux B. (eds). 1996. Biological membranes: A molecular perspective from computation and experiment. Birkhäuser, Boston.

Miao L. Seifert U, Wortis M, Döbereiner H-G 1994. Budding transitions of fluid-bilayer vesicles: The effect of area-difference elasticity. Phys Rev E 49: 5389-5407.

Mouritsen OG. 2005. Life-As a matter of fat: The emerging science of lipidomics. Springer, Heidelberg.

Mouritsen OG. 2010. The liquid-ordered state comes of age. Biochim Biophys Acta 1798: 1286-1288.

Mouritsen OG, Andersen OS, eds. 1998. In search of a new biomembrane model. Biol Skr Dan Vid Selsk 49: 1-214.

Mouritsen OG, Bloom M. 1984. Mattress model of lipidprotein interactions in membranes. Biophys J 46: 141-53.
Mouritsen OG, Jørgensen K. 1994. Dynamical order and disorder in lipid bilayers. Chem Phys Lipids 73: 3-26.

Mouritsen OG, Zuckermann MJ. 2004. What's so special about cholesterol? Lipids 39: 1101-1113.

Nag K, ed. 2008. Membranous interfaces. Wiley, Hoboken, NJ.

Oesterhelt F, Oesterhelt D, Pfeiffer M, Engel A, Gaub HE, Müller DJ. 2000. Unfolding pathways of individual bacteriorhodopsins. Science 288: 143-146.

Patra M. 2005. Lateral pressure profiles in cholesterol-DPPC bilayers. Eur J Biophys 35: 79-88.

Pedersen PJ, Adolph SK, Subramanian AK, Arouri A, Andresen TL, Mouritsen OG, Madsen R, Madsen MW, Peters GH, Clausen MH. 2010. Liposomal formulation of retinoids designed for enzyme triggered release. J Med Chem 53: 3782-3792.

Pencer J, Mills TT, Kucerka N, Nieh MP, Katsaras J. 2007. Small-angle neutron scattering to detect rafts and lipid domains. Methods Mol Biol 398: 231-244.

Perez-Gil J, ed. 2008. BBA special issue: Protein modulation of membrane structure. Biochim Biophys Acta 1778: 15271695.

Pike LJ. 2009. The challenge of lipid rafts. J Lip Res 50: 323-328.

Ponce AM, Vujaskovic Z, Yuan F, Needham D, Dewhirst MW. 2006. Hyperthermia mediated liposomal drug delivery. Int J Hyperth 22: 205-213.

Sabra MC, Mouritsen OG. 1998. Steady-state compartmentalization of lipid membranes by active proteins. Biophys J 74: 745-752.

Sackmann E. 1984. Physical basis for trigger processes and membrane structures. In Biological membranes (ed. Chapman D), pp. 105-143. Academic Press, London.

Sackmann E. 1995. Biological membranes. Architecture and function. In Handbook of biological physics. Structure and dynamics of membranes (ed. Lipowsky R, Sackmann E), Vol 1A, pp. 1-63. Elsevier, Amsterdam.

Seddon JM, Templer RH. 1995. Polymorphism of lipidwater systems. In Handbook of biological physics. Structure and dynamics of membranes (ed. Lipowsky R, Sackmann E), Vol 1A, pp. 97-160. Elsevier, Amsterdam.

Shillcock JC. 2008. Insight or illusion? Seeing inside the cell with mesoscopic simulations. HFSP J 2: 1-6.

Shimshick EJ, McConnell HM. 1973. Lateral phase separation in phospholipid membranes. Biochemistry 12: 2351-2360.

Simons K, Ikonen E. 1997. Functional rafts in cell membranes. Nature 387: 569-72.

Simonsen AC. 2008. Activation of phospholipase A2 by ternary model membranes. Biophys J 94: 966-975.

Simonsen AC, Bagatolli LA. 2004. Structure of spin-coated lipid films and domain formation in supported membranes formed by hydration. Langmuir 20: 9720-9728.

Singer SJ, Nicolson GL. 1972. The fluid mosaic model of the structure of cell membranes. Science 175: 720-731.

Sonne J, Hansen FY, Peters GH. 2005. Methodological problems in pressure profile calculations for lipid bilayers. J Chem Phys 122: 124903. 
Sparr E, Wennerström H. 2001. Responding phospholipid membranes-Interplay between hydration and permeability. Biophys J 81: 1014-1028.

Tamm LK, ed. 2005. Protein-lipid interactions-From membrane domains to cellular networks. Wiley-VCH, Weinheim.

Tanaka M, Sackmann E. 2005. Polymer-supported membranes as models of the cell surface. Nature 437: 656-663.

Thormann E, Dreyer JK, Simonsen AC, Hansen PL, Hansen S, Holmskov U, Mouritsen OG. 2007. Dynamic strength of the interaction between lung surfactant protein $\mathrm{D}$ (SP-D) and saccharide ligands. Biochemistry 46: 12231-12237.

Tuominen EKJ, Wallace CJA, Kinnunen PKJ. 2002. Phospholipid-cytochrome $c$ interaction. Evidence for the extended lipid anchorage. J Biol Chem 277: $8822-8826$.
Turner MS, Sens P, Socci ND. 2005. Nonequilibrium raftlike membrane domains under continuous recycling. Phys Rev Lett 95: 168301.

van Meer G, Voelker DR, Feigenson GW. 2008. Membrane lipids: Where they are and how they behave. Nat Rev Mol Cell Biol 9: 112-124.

Veatch SL, Keller SL. 2003. Separation of liquid phases in giant vesicles of ternary mixtures of phospholipids and cholesterol. Biophys J 85: 3074-3083.

Veatch SL, Polozov IV, Gawrisch K, Keller SL. 2004. Liquid domains in vesicles investigated by NMR and fluorescence microscopy. Biophys J 86: 2910-2922.

Veatch SL, Soubias O, Keller SL, Gawrisch K. 2007. Critical fluctuations in domain-forming lipid mixtures. Proc Natl Acad Sci 104: 17650-17655.

Vist MR, Davis JH. 1990. Phase equilibria of cholesteroldipalmitoyl phosphatidylcholine mixtures: $2 \mathrm{H}$ nuclear magnetic resonance and differential scanning calorimetry. Biochemistry 29: 451-464. 


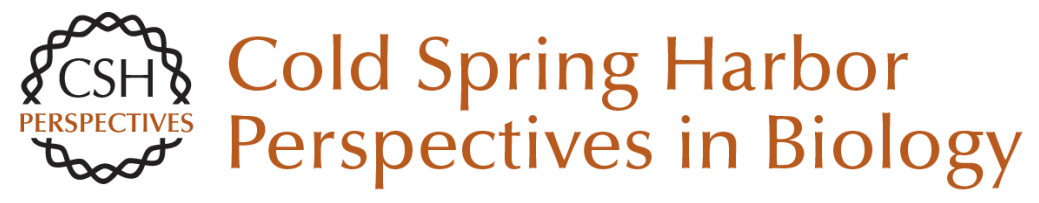

\section{Model Answers to Lipid Membrane Questions}

Ole G. Mouritsen

Cold Spring Harb Perspect Biol 2011; doi: 10.1101/cshperspect.a004622 originally published online May 24, 2011

\section{Subject Collection The Biology of Lipids}

Role of Lipids in Virus Replication Maier Lorizate and Hans-Georg Kräusslich

Model Answers to Lipid Membrane Questions Ole G. Mouritsen

\section{Glycosphingolipid Functions} Clifford A. Lingwood

Regulation of Cholesterol and Fatty Acid Synthesis

Jin Ye and Russell A. DeBose-Boyd

\section{Lipid-Mediated Endocytosis}

Helge Ewers and Ari Helenius

\section{Fluorescence Techniques to Study Lipid \\ Dynamics}

Erdinc Sezgin and Petra Schwille

Lysosomal Lipid Storage Diseases

Heike Schulze and Konrad Sandhoff

\section{Distribution and Functions of Sterols and} Sphingolipids

J. Thomas Hannich, Kyohei Umebayashi and Howard Riezman
Membrane Organization and Lipid Rafts Kai Simons and Julio L. Sampaio

Shotgun Lipidomics on High Resolution Mass

Spectrometers

Dominik Schwudke, Kai Schuhmann, Ronny

Herzog, et al.

Glycosphingolipid Functions Clifford A. Lingwood

Phosphoinositides in Cell Architecture Annette Shewan, Dennis J. Eastburn and Keith Mostov

Synthesis and Biosynthetic Trafficking of Membrane Lipids Tomas Blom, Pentti Somerharju and Elina Ikonen

Lipid Polymorphisms and Membrane Shape Vadim A. Frolov, Anna V. Shnyrova and Joshua Zimmerberg

Specificity of Intramembrane Protein-Lipid Interactions

Francesc-Xabier Contreras, Andreas Max Ernst, Felix Wieland, et al.

Dynamic Transbilayer Lipid Asymmetry Gerrit van Meer

For additional articles in this collection, see http://cshperspectives.cshlp.org/cgi/collection/

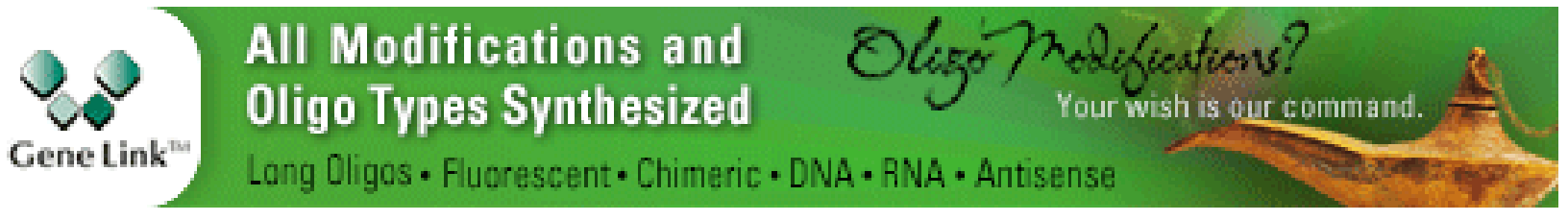

Copyright @ 2011 Cold Spring Harbor Laboratory Press; all rights reserved 